\title{
Article \\ Spectral Broadening in a Continuously Pumped Singly Resonant Second-Harmonic Cavity
}

\author{
Simona Mosca ${ }^{1}$, Tobias Hansson ${ }^{2}$ and Maria Parisi ${ }^{1, *(D)}$ \\ 1 CNR-INO, Istituto Nazionale di Ottica, Via Campi Flegrei 34, I-80078 Pozzuoli, NA, Italy; \\ simona.mosca@ino.cnr.it \\ 2 Department of Physics, Chemistry and Biology, Linköping University, SE-581 83 Linköping, Sweden; \\ tobias.hansson@liu.se \\ * Correspondence: maria.parisi@ino.cnr.it
}

check for updates

Citation: Mosca, S.; Hansson, T.; Parisi, M. Spectral Broadening in a Continuously Pumped Singly Resonant Second-Harmonic Cavity. Appl. Sci. 2021, 11, 7122. https:// doi.org/10.3390/app11157122

Academic Editor: Ruediger

Grunwald

Received: 29 June 2021

Accepted: 30 July 2021

Published: 1 August 2021

Publisher's Note: MDPI stays neutral with regard to jurisdictional claims in published maps and institutional affiliations.

Copyright: (c) 2021 by the authors. Licensee MDPI, Basel, Switzerland. This article is an open access article distributed under the terms and conditions of the Creative Commons Attribution (CC BY) license (https:/ / creativecommons.org/licenses/by/ $4.0 /)$.
Featured Application: The spectral broadening observed in the designed second-order nonlinear optical system is suitable to be miniaturized as a chip-scale multifrequency source for applications in metrology, sensing, and telecommunication.

\begin{abstract}
Optical frequency comb synthesizers with a wide spectral range are an essential tool for many research areas such as spectroscopy, precision metrology, optical communication, and sensing. Recent studies have demonstrated the direct generation of frequency combs, via secondorder processes, that are centered on two different spectral regions separated by an octave. Here, we present the capability of optical quadratic frequency combs for broad-bandwidth spectral emission in unexplored regimes. We consider comb formation under phase-matched conditions in a continuouswave pumped singly resonant second-harmonic cavity, with large intracavity power and control of the detuning over several cavity linewidths. The spectral analysis reveals quite distinctive sidebands that arise far away from the pump, singularly or in a mixed regime together with narrowband frequency combs. Notably, by increasing the input power, the optical frequency lines evolve into widely spaced frequency clusters, and at maximum power, they appear in a wavelength range spanning up to $100 \mathrm{~nm}$. The obtained results demonstrate the capacity of second-order nonlinearities to produce direct comb within a wide range of wavelengths.
\end{abstract}

Keywords: optical frequency combs; quadratic nonlinearity; second-harmonic generation

\section{Introduction}

The increasing demand of metrological and high-resolution spectroscopic measurements drives optical frequency combs (OFCs) synthesizers to be more and more performing in terms of pump power levels, wavelength ranges and chip-scale integration [1,2]. In particular, OFCs act as a frequency ruler and allow to measure optical frequencies with radio frequency accuracy $[3,4]$. A traditional way to generate OFCs is femtosecond mode-locked lasers, their development and applications having been awarded with the 2005 Nobel Prize in physics to Theodor W. Hänsch and John L. Hall [5,6]. Soon thereafter, in 2007, OFCs were demonstrated in microresonators pumped by a continuous-wave (cw) laser, exploiting the third-order nonlinear susceptibility $\chi^{(3)}$, in so called Kerr combs [7,8]. Recent theoretical studies and experimental investigations have demonstrated frequency comb generation also in quadratic nonlinear optical cavities, realizing quadratic frequency combs [9-23]. These sources provide an interesting alternative to traditional comb generation techniques. They offer numerous advantages in terms of low pump power requirements, spectral versatility, and potential for miniaturization of the device [17-23]. Interestingly, they can generate simultaneous combs in different spectral ranges. However, so far, the observed local spectral broadening has been limited. 
A critical point is to extend the spectra of quadratic OFCs to meet the requirements of frequency metrology, molecular spectroscopy, and astronomical applications. Several methods have been developed to expand traditional OFCs to wider spectral regions via spectral broadening in optical fibers [24,25], difference frequency generation schemes [26], and optical parametric oscillators (OPOs) [27,28] and amplifiers [29]. Interestingly, spectral broadening was recently observed in a cw pumped OPO with an additional intracavity crystal, intentionally phase-mismatched for the second-harmonic process [30]. However, the possibility of direct broadband multifrequency emission in a second-order nonlinear cavity, shown here, provides important advantages in terms of device simplicity, size, and efficiency, which are worth exploring. Our system is furthermore attractive, in a long-term perspective, for the miniaturization of quadratic comb synthesizers through the chip-scale realization of photonic circuits [31].

In this work, we present a novel mechanism of spectral broadening of quadratic frequency combs carried out directly in a continuous-wave pumped singly resonant secondharmonic cavity. Most significantly, we observe multifrequency parametric oscillations distant up to $50 \mathrm{~nm}$ from the pump wavelength, and a broad frequency emission intermediate between the pump and the second harmonic. The observed spectral dynamics of the quadratic OFC generation is explained by sideband generation through higher-order modulational instability (MI), induced by the cavity boundary conditions [32]. In the following, we report on the experimental observation of new unexplored regimes in a continuously pumped singly resonant second-harmonic cavity and discuss the results.

\section{Experimental Setup}

The experimental scheme we used is sketched in Figure 1a. A $15 \mathrm{~mm}$ long periodically poled $\mathrm{MgO}: \mathrm{LiNbO}_{3}$ crystal, with a poling period of $\Lambda=6.95 \mu \mathrm{m}$, was placed in a traveling wave bow-tie cavity that was resonant for the pump and with a free spectral range (FSR) of $501 \mathrm{MHz}$. The cavity had a mirror mounted on a piezoelectric actuator for cavity length control and the crystal temperature was actively stabilized by a Peltier element for phase-matching control. The pump source is a narrow linewidth ( $40 \mathrm{kHz}$ over $1 \mathrm{~ms}) \mathrm{cW}$ $\mathrm{Yb}$-doped fiber laser, emitting at $1064 \mathrm{~nm}$. A laser power of a few $\mathrm{mW}$ seeded a $\mathrm{Yb}$-doped fiber amplifier that amplifies it up to $6 \mathrm{~W}$ and pumped the second-harmonic generation (SHG) cavity. We obtained high-power buildup by implementing the cavity using a $99 \%-$ reflectivity plane coupling mirror, a curved mirror with reflectivity $R>99.98 \%$, while the remaining mirrors were high-reflection coated $(\mathrm{R}>99.9 \%)$. The measured cavity resonance full width at half maximum is $1.2 \mathrm{MHz}$ (finesse, 400; Q-factor, $\sim 2 \cdot 10^{8}$ ) when the pump power is $10 \mathrm{~mW}$ (cold cavity). In these conditions, we observed internally pumped $\mathrm{OPO}$ as in [11], but we measured a lower threshold of $20 \mathrm{~mW}$ input pump power. We thermally stabilized the dedicated cavity breadboard by active control to enable robust long-term operation.

We performed active control of the cavity length through frequency offset locking by applying a modified Pound-Drever-Hall (PDH) technique to a few-mW laser beam that was coupled into the SHG cavity in the opposite direction of the pump beam, similarly as reported in [33]. The main advantage of this system is that the control beam does not interfere with the comb generation process because we use a weak probe laser, counterpropagating and orthogonally polarized to the pump beam. The carried-out locking scheme provided control of the cavity length and allowed us to change the resonator-pump frequency detuning $\Delta$ on-demand. 


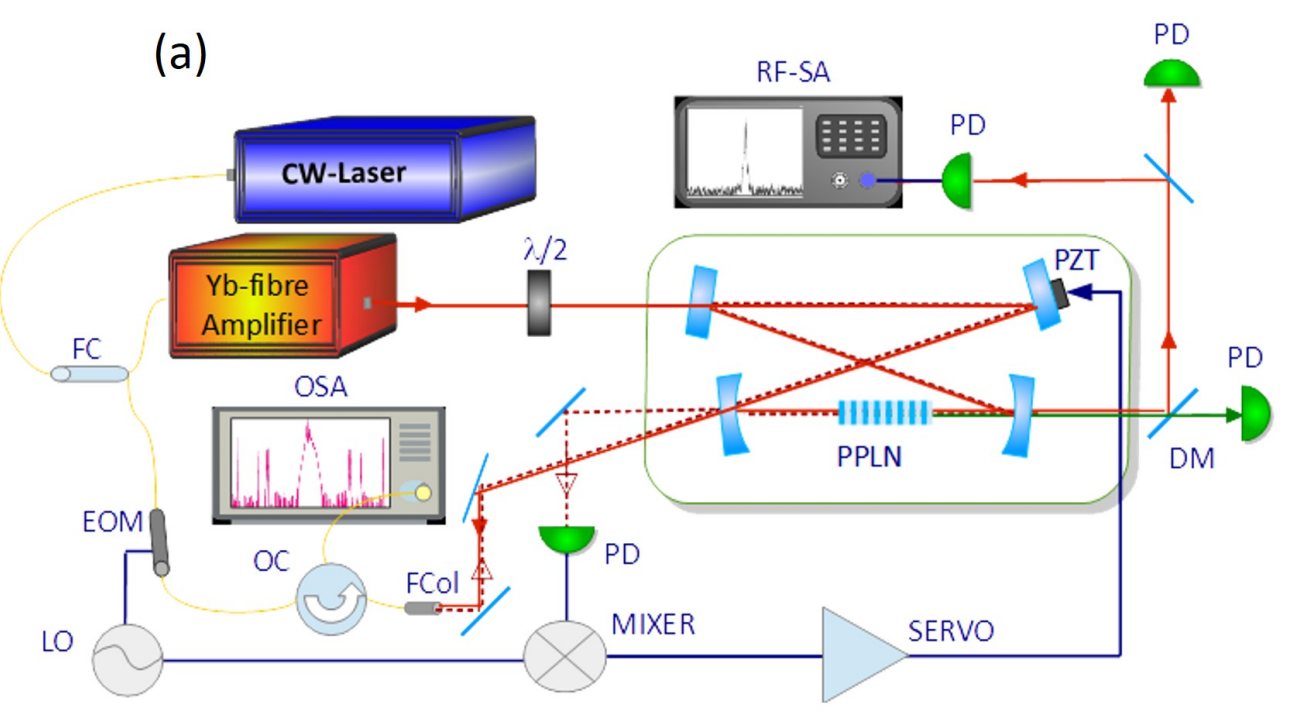

(b)

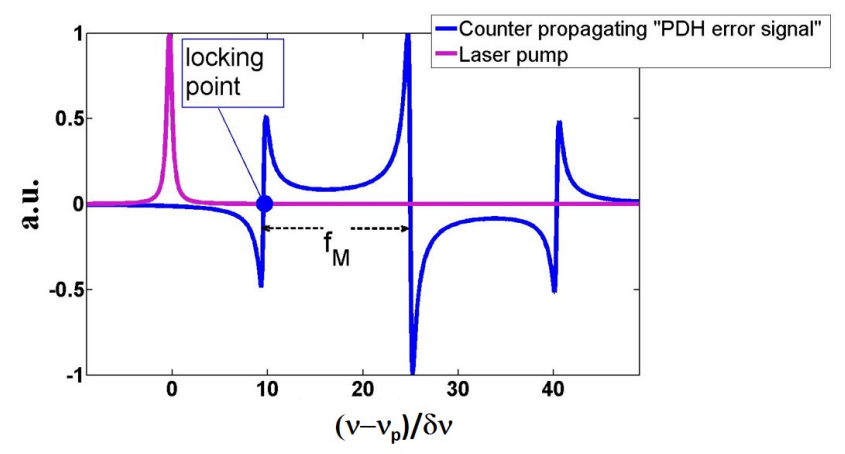

Figure 1. (a) Scheme of the experimental setup for broad-bandwidth comb generation including $\mathrm{cw}$-pumped singly resonant SH cavity with PDH setup for cavity length stabilization. Red and green lines are free-space infrared and optical beams, respectively, yellow are fibers and blue electric cables. Dichroic mirror (DM), electro-optic modulator (EOM), fiber coupler (FC), fiber collimator (FCol), local oscillator (LO), optical circulator (OC), optical spectrum analyzer (OSA), periodically poled $\mathrm{MgO}: \mathrm{LiNbO}_{3}$ crystal (PPLN), piezoelectric actuator (PZT), photodiode (PD), half-wave plate $(\lambda / 2)$, radio-frequency spectrum analyzer (RF-SA); $(\mathbf{b})$ operating principle of the active frequency detuning control where the pump laser beam is free running, while the cavity is locked on the negative sideband frequency of the PDH error signal, obtained by a counter-propagating beam that is orthogonally polarized to the pump.

\section{Experimental Results and Discussion}

Our experimental study reports on the spectral dynamic properties of a cw-pumped singly resonant cavity implemented with tunable cavity length control that utilizes the quasi-phase-matched second-harmonic process. Essentially, the detuning of the optical cavity resonance was stabilized, not to the pump beam, but to a counter-propagating beam, via an adjustable offset sideband locking that it was scanned across the pump frequency to generate emission in various frequency ranges.

The locking point of the PDH servo corresponds to the low-frequency PDH sideband, and the cavity resonance is swept across the optical pump frequency by changing the modulation frequency $\left(f_{M}\right)$, as shown in Figure $1 b$. This scheme allowed us to explore a rich variety of spectral regimes by controlling the detuning over several cavity linewidths. The pump frequency and second-harmonic powers were measured at the resonator output to follow their temporal evolution as the frequency detuning changed. Furthermore, the infrared cavity output was monitored via an optical spectrum analyzer and the inter- 
modal beat notes were detected by a fast photodetector and measured by a radio-frequency spectrum analyzer.

Through suitable changes in the modulation frequency, we expanded the cavity so that the cavity resonance $v_{r}$ could be tuned from higher to lower frequencies across the laser frequency $v_{p}$ (Figure $1 \mathrm{~b}$ ), with the resonance tuned from the blue side of the pump to the red side accordingly. Specifically, we observe that by increasing the cavity length, as $v_{r}$ moves towards $v_{p}$, the onset of thermal effects leads to a measured fringe broadening over several cold cavity linewidths. When $v_{r}$ is lower than $v_{p}$, the intracavity power rapidly decreases and shows a triangular asymmetric fringe shape $[34,35]$. The measured hot-cavity frequency detuning results are shown in Figure 2 for a pump power of $2 \mathrm{~W}$.
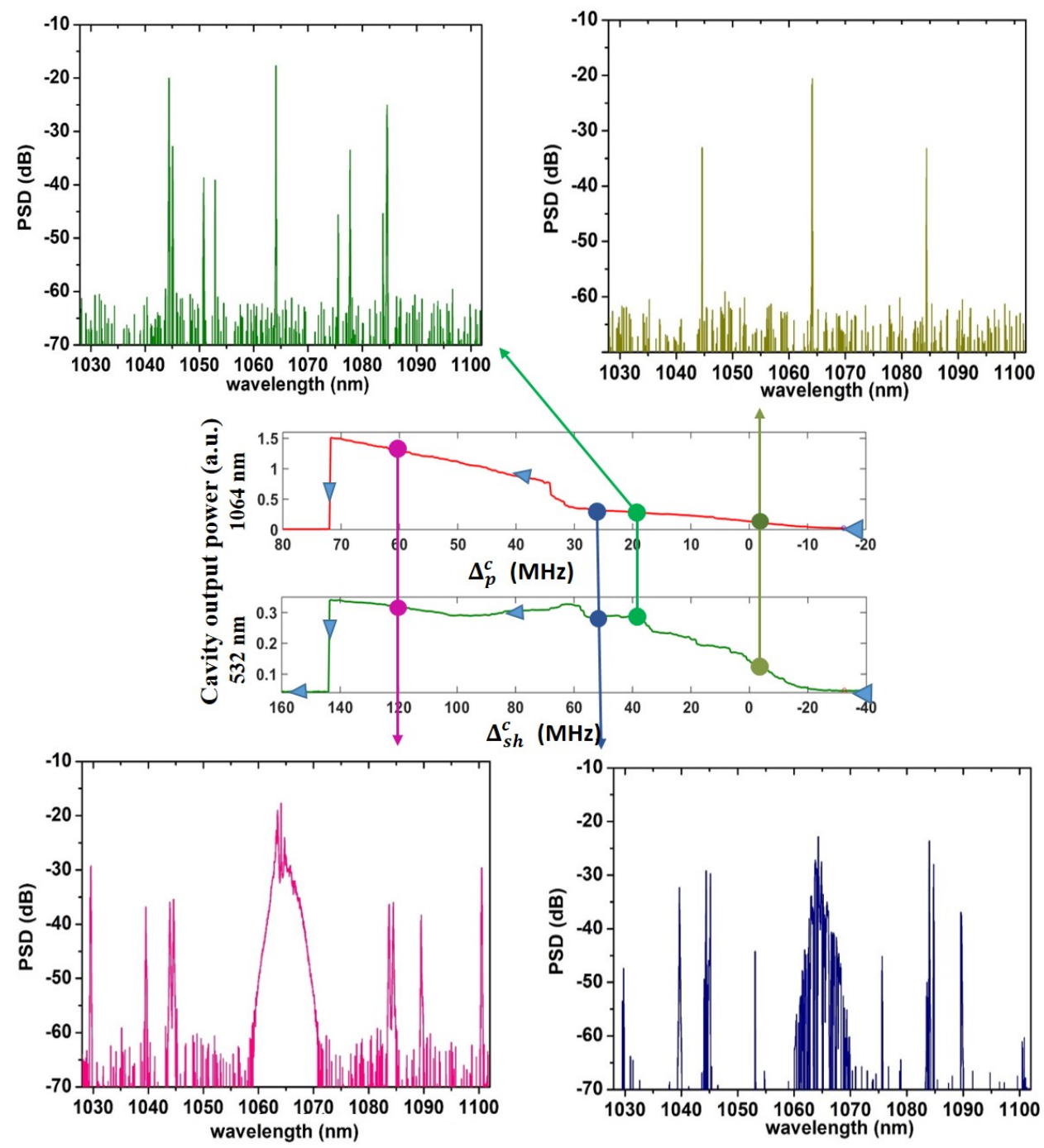

Figure 2. Measured output power when the cavity resonance scans across the pump from smaller to larger frequency. Panels show evolution of the power spectral density (PSD) during the laser scan in four transmission profiles.

Here, we investigate comb formation under an adiabatic cavity length scan with $v_{r}>$ $v_{p}$ and resonator-pump frequency detuning $\Delta=v_{p}-v_{r}<0$ (blue-side). The optothermal dynamics produce a self-stabilizing effect that allows us to record the comb spectra during the cavity length scan. Figure 2 displays the pump and second-harmonic cavity output power as a function of the frequency shift of the cold-cavity frequency detuning $\Delta^{c}$ defined by $\Delta^{c}{ }_{p}=v_{0}-v_{r}$ and $\Delta^{c}{ }_{s h}=2 \cdot \Delta^{c} p$. Here, $v_{0}$ is the cold cavity resonance frequency measured at $10 \mathrm{~mW}$ input power. 
$\Delta^{c}$ can be estimated by measuring the $\mathrm{f}_{M}$ changes, while the effective frequency detuning $\Delta$ is such that $\Delta \leq \Delta^{c}$ under normal operating conditions.

Exploiting changes in the stabilized cavity length, we are able to tune the resonance frequency more than a hundred cold cavity linewidths while keeping the effective frequency detuning negative.

At the onset of the internally pumped parametric oscillation, we observe a single pair of widely detuned sidebands, with a frequency shift of approximately $5 \mathrm{THz}$ (tens of thousands of cavity FSRs). As $|\Delta|$ decreases from the blue side, small frequency comb clusters are generated around the pump and additional sidebands appear at new frequencies.

In order to understand the underlying physics, we attempted to model the temporal and spectral dynamics of the OFC generation. The experimentally observed behavior is found not to be well described by the mean-field model [13] that predicts the formation of MI sidebands to occur only close to the pump. However, there is a qualitative agreement with a two-envelope Ikeda map model $[13,15]$ for the fundamental field $A_{m}$ and the secondharmonic field $B_{m}$

$$
\begin{aligned}
& \frac{\partial A_{m}}{\partial z}=\left[-\frac{\alpha_{c 1}}{2}-i \frac{k_{1}^{\prime \prime}}{2} \frac{\partial^{2}}{\partial \tau^{2}}\right] A_{m}+i \kappa B_{m} A_{m}^{*} e^{-i \Delta k z}, \\
& \frac{\partial B_{m}}{\partial z}=\left[-\frac{\alpha_{c 2}}{2}-\Delta k^{\prime} \frac{\partial}{\partial \tau}-i \frac{k_{2}^{\prime \prime}}{2} \frac{\partial^{2}}{\partial \tau^{2}}\right] B_{m}+i \kappa A_{m}^{2} e^{i \Delta k z},
\end{aligned}
$$

with boundary conditions

$$
A_{m+1}(0)=\sqrt{\theta_{1}} A_{i n}+\sqrt{1-\theta_{1}} e^{-i \delta_{1}} A_{m}(L), \quad B_{m}(0)=0 .
$$

Here, $\mathrm{m}$ is the roundtrip index, the group-velocity dispersion parameters are the same as in [13], viz., $k_{1}^{\prime \prime}=0.234 \mathrm{ps}^{2} / \mathrm{m}$ and $k_{2}^{\prime \prime}=0.714 \mathrm{ps}^{2} / \mathrm{m}$, respectively, $\Delta k^{\prime}=792 \mathrm{ps} / \mathrm{m}$ is the temporal walk-off, $\Delta k=0$ is the phase mismatch, $\kappa=11.41 \mathrm{~W}^{-1 / 2} \mathrm{~m}^{-1}$ is the nonlinear coefficient, $L=15 \mathrm{~mm}$ is the cavity length, $\theta_{1}=\alpha_{c 1} L=\pi / 400$ is the coupling coefficient of the fundamental field, $\alpha_{c 2}=\alpha_{c 1}$ are the absorption losses, while $P_{i n}=\left|A_{\text {in }}\right|^{2}$ is the pump power, and $\delta_{1}=2 \pi k_{1}^{\prime} L \Delta$ is the detuning where $k_{1}^{\prime}$ is the group velocity (i.e., $\delta_{1}=2 \pi$ corresponds to one FSR).

The Ikeda map predicts the formation of higher order instability sidebands due to the cavity boundary conditions [32,36]. Both higher-order modulational (Turing) and parametric (Faraday) instabilities have previously been investigated in the context of cubic Kerr cavity resonators $[37,38]$. The presence of large phase shifts can in particular lead to the formation of so-called Arnold tongues for the periodic parameter domains of the instability [39]. However, the observation of such higher-order instabilities has thus far not been reported in quadratically nonlinear resonators.

Specifically, the simulations show simultaneously quadratic combs generation close to the pump and SH frequencies together with additional sidebands, as shown in Figure 3, although the model fails to predict the intermediate pair of sidebands around 1045 and $1085 \mathrm{~nm}$ (left Figure 3) that are experimentally observed (compare the pink panel of Figure 2).

In order to explain the difference in the observed spectra, we suppose that some residual phase mismatch or additional competing processes could act to perturb the dynamics. A hypothesis could be the presence of a significant cubic nonlinearity. By including a strong simultaneous Kerr nonlinearity in our simulations, we can observe a suppression of the narrowband instability that favors the growth of higher-order sidebands. However, we estimate that the intensity of the beam is too low for the cubic nonlinearity to have an appreciable effect and that it would require a more focused beam with higher intensity to make the magnitudes of the cubic and quadratic nonlinearities comparable.

Hereafter, we focus our investigations on the maximum achievable wavelength broadening for high $\Delta^{c}$ values, i.e., in the region where the pump laser is less blue detuned (Figure 4). We report spectral broadening for pump powers up to $6 \mathrm{~W}$. For $0.3 \mathrm{~W}, 0.6 \mathrm{~W}$, 
and $1 \mathrm{~W}$, the acquired spectra correspond to $\Delta^{c}$ values of 9,15 , and $30 \mathrm{MHz}$, respectively. For larger powers, we used a thermally induced self-locking of the optical cavity [40], and the $\Delta^{c}$ values are, therefore, not measured exactly. Increasing the input power, the parametric sidebands arising far from the pump evolve into "clustered" optical frequency combs, and at the maximum power available, they extend over a wavelength range spanning up to $100 \mathrm{~nm}$.
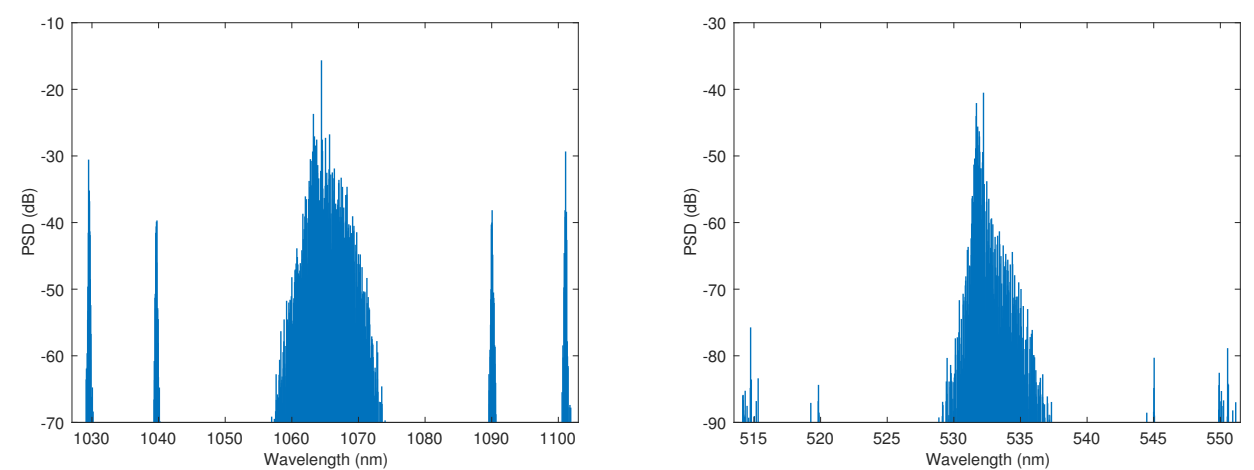

Figure 3. Ikeda map simulation with $P_{\text {in }}=2 \mathrm{~W}$ and $\delta_{1}=-0.0392$ showing comb generation similar to the pink panel in Figure 2. Panels show fundamental (left) and second harmonic spectra (right).
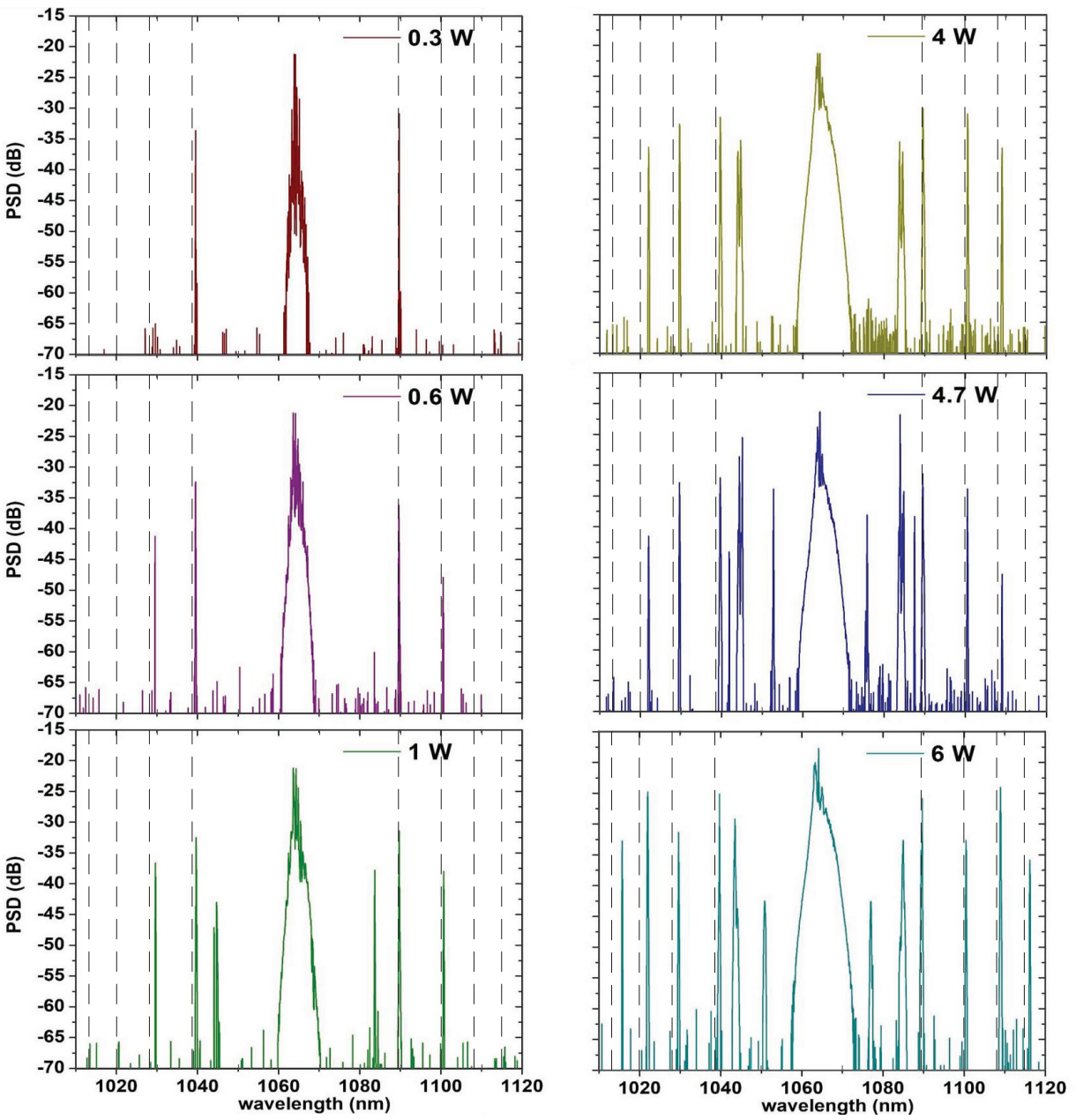

Figure 4. Optical spectral power around the fundamental mode for $0.3 \mathrm{~W}, 0.6 \mathrm{~W}, 1 \mathrm{~W}, 4 \mathrm{~W}, 4.7 \mathrm{~W}$, and $6 \mathrm{~W}$ of input power. Dashed lines show predicted sideband positions. 
The position of these sidebands can be estimated from the phase-matching condition $\delta_{1}-\left(k_{1}^{\prime \prime} L / 2\right) \Omega^{2}=m \pi$, where we neglect nonlinear phase shifts. We find that the sideband positions are approximately as follows:

$$
\Omega_{m} \approx \sqrt{\frac{2}{k_{1}^{\prime \prime} L}\left(\delta_{1}+m \pi\right)}, \quad m=0,1,2, \ldots
$$

with the pump detuning $\delta_{1} \approx 0$ and where the order $m=0$ corresponds to the ordinary MI. The predicted positions of the $m=[1,2,3,4]$ sidebands are shown as dashed lines in Figure 4. The position of the first higher-order $m=1$ sideband is approximately 6.7 $\mathrm{THz}$, which is in good agreement with the measured frequency shift. Increasing the pump power leads to the generation of additional sidebands with even larger frequency shifts that approximately match with the higher-order positions of the model. This is a strong indication that the sidebands originate from boundary conditions induced MI. Sidebands with smaller frequency shifts are also observed at higher pump powers, but these intermediate sidebands are neither predicted by Equation (4) nor observed in the Ikeda map simulations.

Furthermore, we observe that various nonlinear cascaded processes lead to wavelength emission that extends not just around the pump but also around $840 \mathrm{~nm}$, a spectral range not previously observed. In Figure 5, we report spectral measurements in the maximum wavelength range of the optical spectrum analyzer, together with the radio-frequency (RF) spectrum of the pump frequency recorded at $6 \mathrm{~W}$ of input power. The RF spectrum reveals a frequency comb structure with an adjacent peak separation close to $501 \mathrm{MHz}$, corresponding to the FSR of the cavity.

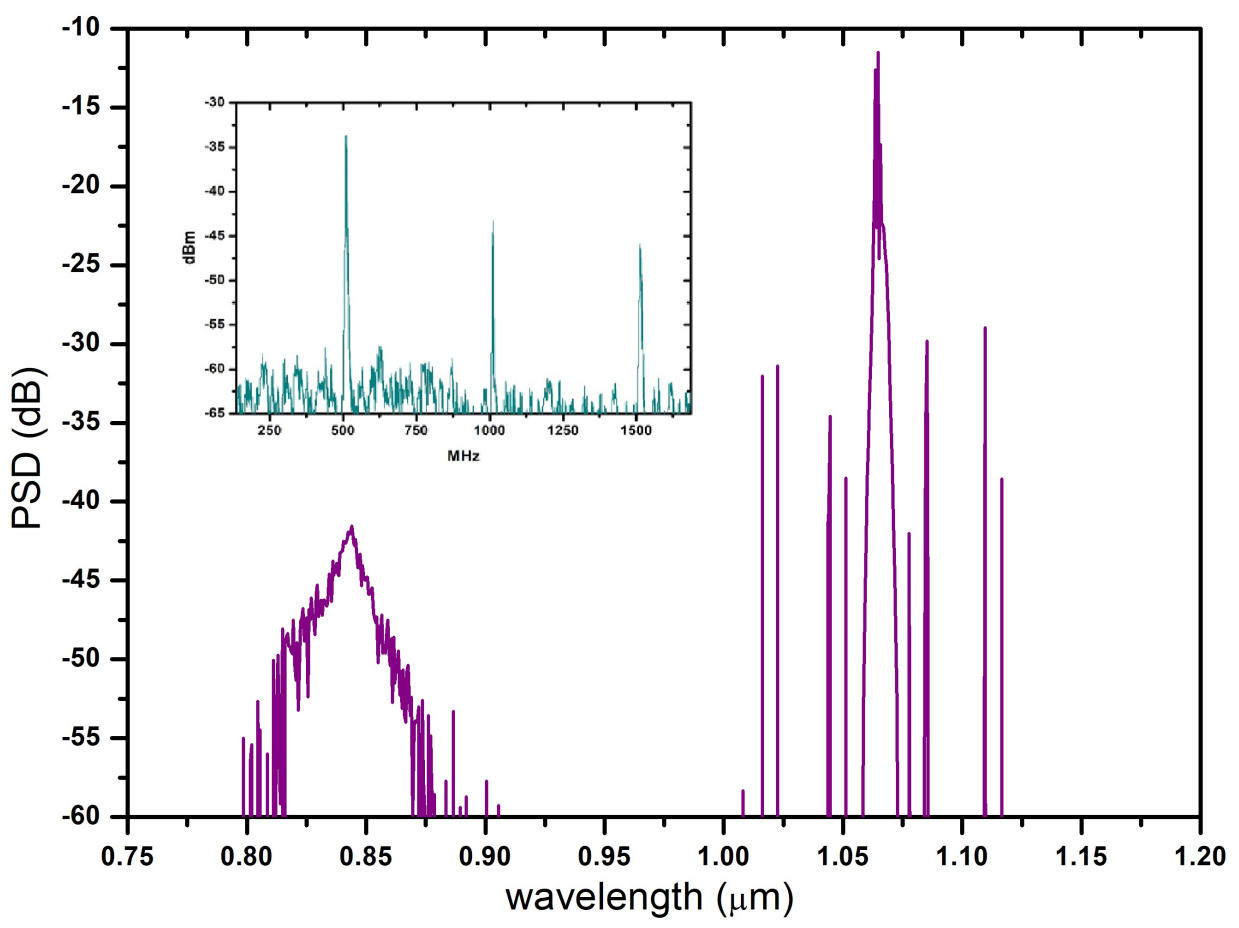

Figure 5. Measured wavelength emission in the maximum range of the optical spectrum analyzer with $6 \mathrm{~W}$ input power and in inset the radio frequency spectrum.

Additional processes can occur since the QPM grating phase-matches not only the primary SHG process, but also other combinations of waves. For example, using the Sellmeier equation for $\mathrm{LiNbO} 3$, we find the possibility of having additional sum-frequency generation and OPO processes [12]. In fact, the blue side of the $\mathrm{SH}$ supports nondegenerate OPO. Any wavelength $\lambda_{3}<532 \mathrm{~nm}$ will satisfy the energy conservation $\omega_{3}=\omega_{1}+\omega_{2}$ 
and phase-matching condition $k_{3}-k_{1}-k_{2}+k_{Q}=0$ (with $k_{i}=2 \pi / \lambda_{i}, i=1,2,3$ and $\left.k_{Q}=2 \pi / \Lambda\right)$, for some combination of frequencies. Additional three-wave mixing processes can moreover be phase-matched by multiple order wavevectors of the QPM grating.

Unfortunately, the dynamics of these combs are outside the range of applicability of current models $[13,15,41]$. Their numerical simulation would require the use of a single envelope equation model or the development of a new multi-envelope Ikeda map model that is beyond the scope of the current investigation and will be the subject of upcoming works. Although our hypotheses cannot provide a complete clarification of the growth of the MI sidebands both close and far away from the pump, these theoretical cues are an interesting starting point for future investigations.

The present study shows the appearance of novel spectral broadening effects in $\chi^{(2)}$ frequency combs. Multifrequency comb emissions are expected to play a fundamental role for numerous applications and the development of improved theoretical models, and new investigations will be an essential key for the progression of knowledge.

\title{
4. Conclusions
}

In conclusion, we show that new nonlinear regimes occur with high power buildup in a cw-pumped SHG cavity. By controlling the detuning over several cavity linewidths, we investigate the system dynamic and observe new comb regimes that extend over a broad spectral range. Most significantly, the obtained results illustrate the onset of sideband oscillations far away from the main quadratic frequency comb around the pump wavelength. Exploiting the wide spectral coverage and the coherent multifrequency emission in quadratic nonlinear cavities it is possible to enable a dramatic extension of applications for high-resolution spectroscopy, optical telecommunication, and quantum photonic sources. The studied quadratic nonlinear system is suitable to be miniaturized by replacing the optical bench-top mounting system with a quadratic nonlinear waveguide resonator. For instance, a new on-chip synthesizer could be based on the previously investigated design [17], which utilizes the AlGaAs platform. AlGaAs, with its efficient $\chi^{(2)}$ nonlinearity, can operate with relatively low pump power, and its compatibility with the CMOS industry makes it a promising candidate to develop a waveguide resonator for multifrequency integrated sources on small photonic chips. Furthermore, our measurements may open the way for future theoretical and experimental investigations on the quantum correlations between the generated beams.

Author Contributions: Management, M.P.; setup implementation, measurements and data analysis, S.M. and M.P.; theoretical study, T.H.; results discussions, all; writing—original draft preparation, S.M. and M.P.; writing-review and editing, all; supervision, M.P. All authors have read and agreed to the published version of the manuscript.

Funding: This research was supported in part by European Union's Horizon 2020 research and innovation program (Qombs Project, FET Flagship on Quantum Technologies Grant. No. 820419). T.H. acknowledges funding from the Swedish Research Council (Vetenskapsrådet, Grant. No. 2017-05309).

Acknowledgments: The authors gratefully acknowledge suggestions and stimulating discussions from Paolo De Natale and Pasquale Maddaloni. They thank G. Notariale for technical support.

Conflicts of Interest: The authors declare no conflict of interest.

\author{
Abbreviations \\ The following abbreviations are used in this manuscript: \\ $\mathrm{cw}$ Continuous wave \\ $\mathrm{f}_{M} \quad$ Frequency modulation \\ FSR Free spectral range \\ MI Modulational instability \\ OFC Optical frequency comb \\ OPO Optical parametric oscillator \\ PD Photodetector
}


PDH Pound Drever Hall

PSD Power spectral density

RF Radio frequency

$\mathrm{SH}$ Second harmonic

SHG Second harmonic generation

\section{References}

1. Picqué, N.; Hänsch, T.W. Frequency comb spectroscopy. Nat. Photonics 2019, 13, 146-157. [CrossRef]

2. Udem, T.; Holzwarth, R.; Hänsch, T.W. Optical frequency metrology. Nature 2002, 416, 233-237. [CrossRef] [PubMed]

3. Diddams, S.A.; Udem, T.; Bergquist, J.C.; Curtis, E.A.; Drullinger, R.E.; Hollberg, L.; Itano, W.M.; Lee, W.D.; Oates, C.W.; Vogel, K.R.; et al. An Optical Clock Based on a Single Trapped 199Hg+ Ion. Science 2001, 293, 825-828. [CrossRef] [PubMed]

4. Ludlow, A.D.; Boyd, M.M.; Ye, J.; Peik, E.; Schmidt, P.O. Optical atomic clocks. Rev. Mod. Phys. 2015, 87, 637. [CrossRef]

5. Hall, J.L. Nobel Lecture: Defining and measuring optical frequencies. Rev. Mod. Phys. 2006, 78, 1279-1295. [CrossRef]

6. Hänsch, T.W. Nobel Lecture: Passion for precision. Rev. Mod. Phys. 2006, 78, 1297-1309. [CrossRef]

7. Del'Haye, P.; Schliesser, A.; Arcizet, O.; Wilken, T.; Holzwarth, R.; Kippenberg, T.J. Optical frequency comb generation from a monolithic microresonator. Nature 2007, 450, 1214-1217. [CrossRef]

8. Kippenberg, T.J.; Holzwarth, R.; Diddams, S.A. Microresonator based optical frequency combs. Science 2011, 332, 555-559. [CrossRef]

9. Ulvila, V.; Phillips, C.R.; Halonen, L.; Vainio, M. Frequency comb generation by a continuous-wave-pumped optical parametric oscillator based on cascading quadratic nonlinearities. Opt. Lett. 2013, 38, 4281. [CrossRef]

10. Ulvila, V.; Phillips, C.R.; Halonen, L.; Vainio, M. High-power mid-infrared frequency comb from a continuous-wave-pumped bulk optical parametric oscillator. Opt. Express 2014, 22 10535. [CrossRef]

11. Ricciardi, I.; Mosca, S.; Parisi, M.; Maddaloni, P.; Santamaria, L.; De Natale, P.; De Rosa, M. Frequency comb generation in quadratic nonlinear media. Phys. Rev. A 2015, 91, 063839. [CrossRef]

12. Mosca, S.; Ricciardi, I.; Parisi, M.; Maddaloni, P.; Santamaria, L.; De Natale, P.; De Rosa, M. Direct generation of optical frequency combs in $\chi^{(2)}$ nonlinear cavities. Nanophotonics 2016, 5, 316-331. [CrossRef]

13. Leo, F.; Hansson, T.; Ricciardi, I.; De Rosa, M.; Coen, S.; Wabnitz, S.; Erkintalo, M. Walk-off-induced modulation instability, temporal pattern formation, and frequency comb generation in cavity-enhanced second-harmonic generation. Phys. Rev. Lett. 2016, 116, 033901. [CrossRef] [PubMed]

14. Hansson, T.; Leo, F.; Erkintalo, M.; Anthony, J.; Coen, S.; Ricciardi, I.; De Rosa, M.; Wabnitz, S. Single envelope equation modeling of multi-octave comb arrays in microresonators with quadratic and cubic nonlinearities. J. Opt. Soc. Am. B 2016, 33, 1207-1215. [CrossRef]

15. Hansson, T.; Leo, F.; Erkintalo, M.; Coen, S.; Ricciardi, I.; De Rosa, M.; Wabnitz, S. Singly resonant second-harmonic-generation frequency combs. Phys. Rev. A 2017, 95, 013805. [CrossRef]

16. Leo, F.; Hansson, T.; Ricciardi, I.; De Rosa, M.; Coen, S.; Wabnitz, S.; Erkintalo, M. Frequency-comb formation in doubly resonant second-harmonic generation. Phys. Rev. A 2016, 93, 043831. [CrossRef]

17. Parisi, M.; Morais, N.; Ricciardi, I.; Mosca, S.; Hansson, T.; Wabnitz, S.; Leo, G.; De Rosa, M. AlGaAs waveguide microresonators for efficient generation of quadratic frequency combs. J. Opt. Soc. Am. B 2017, 34, 1842-1847. [CrossRef]

18. Mosca, S.; Parisi, M.; Ricciardi, I.; Leo, F.; Hansson, T.; Wabnitz, S.; Erkintalo, M.; Maddaloni, P.; Santamaria, L.; De Natale, P.; et al. Modulation Instability Induced Frequency Comb Generation in a Continuously Pumped Optical Parametric Oscillator. Phys. Rev. Lett. 2018, 121, 093903. [CrossRef] [PubMed]

19. Ikuta, R.; Asano, M.; Tani, R.; Yamamoto, T.; Imoto, N. Frequency comb generation in a quadratic nonlinear waveguide resonator. Opt. Express $2018,26,15551-15558$. [CrossRef]

20. Guillemé, P.; Vecchi, C.; Ciret, C.; Castellan, C; Signorini, S.; Ghulinyan, M.; Bernard, M.; Parisi, M.; Pavesi L. Modeling of quasi-phase-matched cavity-enhanced second-harmonic generation. Appl. Sci. 2020, 10, 9047. [CrossRef]

21. Stefszky, M.; Ulvila, V.; Abdallah, Z.; Silberhorn, C.; Vainio, M. Towards optical-frequency-comb generation in continuous-wavepumped titanium-indiffused lithium-niobate waveguide resonators. Phys. Rev. A 2018, 98, 053850. [CrossRef]

22. Hendry, I.; Trainor, L.S.; Xu, Y.; Coen, S.; Murdoch, S.G.; Schwefel, H.G.L.; Erkintalo, M. Experimental observation of internallypumped parametric oscillation and quadratic comb generation in a $\chi^{(2)}$ whispering-gallery-modemicroresonator. Opt. Lett. 2020, 45, 1204-1207. [CrossRef]

23. Szabados, J.; Puzyrev, D.N.; Minet, Y.; Reis, L.; Buse, K.; Villois, A.; Skryabin, D.V.; Breunig, I. Frequency comb generation via cascaded second-order nonlinearities in microresonators. Phys. Rev. Lett. 2020, 124, 203902. [CrossRef]

24. Xia, C.; Kumar, M.; Kulkarni, O.P.; Islam, M.N.; Terry, F.L.; Freeman, M.J.; Poulain, M.; Mazé, G. Midinfrared supercontinuum generation to 4.5 num in ZBLAN fluoride fibers by nanosecond diode pumping. Opt. Lett. 2006, 31, 2553-2555. [CrossRef]

25. Mandon, J.; Sorokin, E.; Sorokina, I.T.; Guelachvili, G.; Picqué, N. Supercontinua for high-resolution absorption multiplex infrared spectroscopy. Opt. Lett. 2008, 33, 285-287. [CrossRef]

26. Erny, C.; Moutzouris, K.; Biegert, J.; Kühlke, D.; Adler, F.; Leitenstorfer, A.; Keller, U. Mid-infrared differencefrequency generation of ultrashort pulses tunable between 3.2 and $4.8 \mu \mathrm{m}$ from a compact fiber source. Opt. Lett. 2007, 32, 1138-1140. [CrossRef] 
27. Sun, J.H.; Gale, B.J.S.; Reid, D.T. Composite frequency comb spanning 0.4-2.4 $\mu \mathrm{m}$ from a phase-controlled femtosecond Ti:sapphire laser and synchronously pumped optical parametric oscillator. Opt. Lett. 2007, 32, 1414-1416. [CrossRef]

28. Adler, F.; Cossel, K.C.; Thorpe, M.J.; Hartl, I.; Fermann, M.E.; Ye, J. Phase-stabilized, 1.5 W frequency comb at $2.8-4.8 \mu \mathrm{m}$. Opt. Lett. 2009, 34, 1330-1332. [CrossRef]

29. Brida, D.; Manzoni, C.; Cirmi, G.; Marangoni, M.; De Silvestri, S.; Cerullo, G. Generation of broadband midinfrared pulses from an optical parametric amplifier. Opt. Express 2007, 15, 15035-15040. [CrossRef] [PubMed]

30. Ulvila, V.; Vainio, M. Experimental study of the effect of phase mismatch on a CW-pumped cascaded quadratic nonlinear frequency comb. J. Phys. Photonics 2020, 2, 034006. [CrossRef]

31. Dietrich, C.P.; Fiore, A.; Thompson, M.G.; Kamp, M.; Höfling, S. GaAs integrated quantum photonics: Towards compact and multifunctional quantum photonic integrated circuits. Laser Photonics Rev. 2016, 10, 870-894. [CrossRef]

32. Coen, S.; Haelterman, M. Modulational Instability Induced by Cavity Boundary Conditions in a Normally Dispersive Optical Fiber. Phys. Rev. Lett. 1997, 79, 4139. [CrossRef]

33. Ricciardi, I.; Mosca, S.; Parisi, M.; Maddaloni, P.; De Natale, P.; Leo, F.; Hansson, T.; Arie, A.; Wabnitz, S.; De Rosa, M. Experimental Observation of Optical Frequency Combs in Doubly Resonant Second Harmonic Generation. In Proceedings of the Conference on Lasers and Electro-Optics Europe and European Quantum Electronics Conference, Munich, Germany, $23-27$ June 2019.

34. Ricciardi, I.; De Rosa, M.; Rocco, A.; Ferraro, P.; De Natale, P. Cavity-enhanced generation of 6 W cw second-harmonic power at $532 \mathrm{~nm}$ in periodically-poled $\mathrm{MgO}: \mathrm{LiTaO}_{3}$. Opt. Express 2010, 18, 10985. [CrossRef]

35. Le Targat, R.; Zondy, J.-J.; Lemonde, P. 75\%-Efficiency blue generation from an intracavity PPKTP frequency doubler. Opt. Commun. 2005, 247, 471-481. [CrossRef]

36. McLaughlin, D.W.; Moloney, J.V.; Newell, A.C. New class of instabilities in passive optical cavities. Phys. Rev. Lett. 1985, 54, 681. [CrossRef]

37. Staliunas, K.; Hang, C.; Konotop, V.V. Parametric patterns in optical fiber ring nonlinear resonators. Phys. Rev. A 2013, 88, 023846. [CrossRef]

38. Copie, F.; Conforti, M.; Kudlinski, A.; Mussot, A.; Trillo, S. Competing Turing and Faraday Instabilities in Longitudinally Modulated Passive Resonators. Phys. Rev. Lett. 2016, 116, 143901. [CrossRef]

39. Skryabin, D.V.; Fan, Z.; Villois, A.; Puzyrev, D.N. Threshold of complexity and Arnold tongues in Kerr-ring microresonators. Phys. Rev. A 2021, 103, L011502. [CrossRef]

40. Dube, P.; Ma, L.S.; Ye, J.; Jungner, P.; Hall, J.L.Thermally induced self-locking of an optical cavity by overtone absorption in acetylene gas. J. Opt. Soc. Am. B 1996, 13, 9. [CrossRef]

41. Mas Arabí, C.; Parra-Rivas, P.; Ciret, C.; Gorza, S.P.; Leo, F. Modeling of quasi-phase-matched cavity-enhanced second-harmonic generation. Phys. Rev. A 2020, 101, 043818. [CrossRef] 